\title{
The role of the Internet both as an ancient agora and a French café for the humanities
}

\author{
Dimitrios Theotokis and Georgios Gyftodimos \\ University of Athens, Department of Informatics \\ Panepistimiopolis, Ilissia 157 71, Athens Greece \\ Email:\{dtheo,geogyf\}@di.uoa.gr
}

\begin{abstract}
The role of the Internet in today's information world can be considered as one of an agora, a French pre-revolution café, or simply an unlimited source of information available to everyone on request. The beneficiaries of this information availability are many. Academic issues as well as everyday discussions may make use of the world wide web to promote debates, raise issues of interest, put forward concepts and ideas, and generally contribute to the proliferation of knowledge even at the most remote location on the globe either as a blue ribbon medium or as a moderated discussion forum. This increasing information availability, on the one hand, and demand, on the other, pose a very important question: what should computer scientists do to make this worldwide spread of information not only available, but also and most importantly, useful to its recipients?
\end{abstract}

\section{INTRODUCTION}

The Internet's rapid evolution has challenged, among other issues, many of the traditional approaches regarding the proliferation of knowledge, the availability of up-to-date information as well as the means for expressing one's own personal views and actively participating in an exchange of both information and knowledge. Frontiers are being bypassed and information is becoming available to everyone to seek and acquire.

Current technologies make it possible to transform the availability of such information and its subsequent ease of manipulation into a learning process ${ }^{1}$. A learning process that encounters no barriers, physical or logical, is available on 
request whenever and wherever such a need arises, can be tailored to meet its recipients needs and, above all, is subject to critical thinking for evaluation.

The Internet, by eliminating distance and physical frontiers, tends to become the forum where ideas are exchanged, cultures are viewed from different perspectives, issues are debated, and new concepts are promoted but, most importantly, people are brought together to form a multinational, frontier-less society that shares a common ideal: information exchange and knowledge proliferation.

The Internet's exclusive role in the 1980s, that of a scientific forum, is rapidly being overtaken by a wider, holistic and multiple perspective that renders its role to one of a means to an end rather than an end in itself. The end is the delivery of information, and its consequent proliferation around the world, that enables end users to become easily updated on developments in their field of study or interest.

Viewed from this perspective, the Internet leads to a number of important issues that require thorough thinking when addressed, such as:

- what security issues arise that need to be addressed?

- how can the validity of information be guaranteed?

- what are the social and political agendas that need to be addressed?

- how are controversial issues dealt with?

- how can this information flood be transformed into a knowledge acquisition mechanism that would not only further the epistemic aspects of the domains it addresses but will also reach people who are at present unable to obtain a broad perspective on their area of interest?

- how can technological developments, present and future, contribute in the better use of existing information pools?

- to what degree will this emerging technology change one's own contribution to society and, as a result, change society itself?

This analysis addresses the proliferation of knowledge and information that stems from existing information sources over the world wide web as well as the roles that the participants of this forum can play in the generation, formation, and exchange of such information and with respect to the humanities. The term humanities encapsulates a wide number of disciplines and aspects of human life. The authors, however, focus on the aspects of human culture, with particular reference to literature, language, history, and philosophy, areas where the Internet can play a very important role yet to be explored.

\section{POSSIBLE ROLES OF THE INTERNET}

So far, the Internet has been considered as the computer people medium for information exchange, a notion very well nursed by the obscurity of Internet access utilities such as ftp and ftam and the painstaking efforts that users had to make in using the Internet. The technological boom and, in particular, that associated with the world wide web, as currently experienced, diffuses this perspective in light of the user-oriented but not necessarily expert-oriented philosophy that has been adopted. Strange and confusing procedures are substituted by friendly graphical network browsers where information is presented in a polymorphic manner by means of video, sound, text, and iconic forms. Its users are no longer expected to 
be computer literate gurus in order to make use of this new born, yet rapidly evolving service. Instead, anyone with the bare minimum of technology can be part of this international forum of communication ${ }^{2}$.

What is, however, the implication of more and more people having access to the same information, being able to communicate among themselves, exchanging their views and ideas, debating key issues, expressing beliefs and, in general, participating in this new found, frontier-less society? One must pause for an instance and draw two analogies of what the Internet can be perceived to be: an agora, on the one hand, and a French café, on the other.

\section{INTERNET: THE WORLDWIDE AGORA}

In the ancient state of Athens, the agora was the place where philosophical discussions, debates, and teaching took place. It was where Socrates and Plato, Demosthenes and Solon, among others, in their broad thinking, fought for their ideas, advanced philosophy, presented their views, and set the foundations for the humanities as we know them today.

In a similar way, today's agora, the electronic one, is not surrounded by pillars that overlook the Acropolis of Athens but is instead located in one's office, home, or workplace where it brings together people from all over the world, giving them the opportunity to participate in an open forum of thought exchange. Ideas ranging from poetry to archaeology and from sociology to pure science all, under the umbrella of the electronic agora, the world wide web, become available to anyone to acquire. Consequently, what used to be the privilege of the few is now available to everyone, for everyone to enjoy, contribute to, and obtain.

Web browsers, the medium through which the agora is realized, provide the means for both listeners and speakers to express their views, opinions, and concerns regarding current affairs and other matters.

Under this perspective, the Internet becomes an utterance for free speech and opinion proliferation, ensuring full freedom of expression for the speaker. For the listener, it provides capabilities of unrestricted and unrestrained searching so that she can choose the source of information she requires, form opinions by processing the information, and possibly become a speaker for or against that information.

The role of the participant as a speaker or listener may change in the web realization of the agora, in the same way it did in the ancient agora. This dynamic adaptation of a participant's role within the context of the agora is what makes the difference between the speaker and listener in broadcasting, and author and reader in journals and newspapers (where these roles depend on limitations imposed by the mediums themselves as well as by political and financial implications). Hereafter, the term speaker is used to refer to the person who addresses world wide web users, while the term listener refers to the recipients of the speaker's communication.

Listeners can dynamically locate, choose, and evaluate the information they require. They can even devote the amount of time they consider appropriate in doing so. In this way, they can process the information they receive, manage it, and even particularize it to the extent they consider useful. Their participation can lead them to reform current opinions and beliefs regarding a matter that has been 
debated. This is a significant perspective when taking into account the amount of information available and its diversity both in content and context.

Through the use of world wide web browsers listeners can, if considered appropriate, follow the interconnections related to the information currently observed whether provided by the speaker or not. As a result, listeners form their own criteria for evaluating the information provided by the speaker.

Browsers also support the user's role as a speaker. The Internet's immediacy brings together the speaker with her audience through their common interests, without the need for complex and expensive media. Users' interest and participation in such a communication may be fundamentally different. In its simplest form, this communication is a thorough observation of what is taking place around the world, like reading a worldwide newspaper that provides immediate and uncensored information. The information found in this newspaper can be traced to its author who can be reached and her views debated at either a public or a private level. At its highest level of complexity, it can be considered as participation in an interactive process where the listener aims to learn from knowledgeable speakers in terms of questions and observations. In this sense, the speaker's address to her audience is not in vain, like a television programme. On the contrary, it must be seen as feedback that originates from the listeners.

The provision of knowledge or opinion that the knowledgeable person has may or may not be filtered, censored or red taped. It is up to the end user, the listener, to decide what to do, when the validity of information is of extreme importance and needs to be examined by experts (for example, conferences on the world wide web, listservers) or in cases where the information provided generates an overflow which in turn results in misinformation.

Although any domain of human interest can be served by the Internet's role as an agora, there is a strong justification for focusing on the field of the humanities because of their relationship to everyone's life. Moreover, the humanities have a lot to gain from the use of the Internet as an agora podium:

- Local information. Even if it were possible to obtain it from all over the world, it would generate mountains of non-accessible data due to its volume, hence rendering it worthless.

- Truths and facts. These fundamental parts of a listener's life may deliberately or not be omitted or infringed. If this is the case listeners, by becoming speakers, can inform the world of such omissions and infringements. Moreover, the feedback generated is useful for the speaker who can use it to motivate other listeners to participate.

Specific issues that rely heavily on the Internet's role as an agora are:

- Presentation by the speaker of particular topics with specific interest that concern, among other things, ethnological and folklore issues. Some listeners may be aware of unknown details and sides that they can contribute to with their experience. Consequently, listeners become a source of reliable and crossreferenced information. Such information becomes more important if placed in a broader frame of collected elements which the listener can abstract or compose.

- The performance of local music by local people who have the feel and knowledge of local instruments, not due to some specific training but through the deep and undetermined knowledge obtained by close and continuous interaction with their environment (family or community). Such knowledge 
would be impossible to formalize in the narrow space that general study provides. Such presentations could, in relation to the specific events when they are used or via the emotions they carry, provide insight to hidden aspects of a way of life.

- Description of historical facts, like archaeological findings from the area where a person lives. Consequently, the individual possesses a broader knowledge regarding these facts even if that knowledge is contained in tales passed down from father to son. Such elements are too small to be studied or, in some cases, are unknown and are lost forever as time passes.

- Minorities, local, national or international can promote their positions and make audiences aware of and, at the same time, sensitive to the facts that concern them more easily than by taking an official protest through the proper channels to which they may not even have access. The availability of such information is of great value since, on the one hand, it becomes the vehicle that transports worldwide problems and, on the other hand, it helps listeners to review or to reform their attitudes, philosophical positions, viewpoints, morals, and emotions by raising problems that exist outside the listener's prejudiced world.

From this perspective, the Internet can be seen as the place where speakers with different opinions regarding the same matter may address an audience whose role can be altered flexibly so as to adopt eventually that of a speaker. The Internet's characteristics range from its simple role as a source of information from which users make a selection based on what interests them and where, at the same time, they are free to manipulate this information to the degree they wish, to the socially and particularly complex manner in which they can actively and consciously participate in a society. Thus, the agora can play a significant role in the evolution of society. Such knowledge is particularly useful in the humanities due to both the importance that the area gains through this frontier-less concept and the discharge of priorities that stem from estimations of national importance that adulterate the information.

Listeners' active participation differentiates their role from the classic one in broadcasting, where no answers can be given or remarks made to the speaker and where the conversation time is strictly limited to that of the speaker. It also differs from the newspaper readers' role, since the latter can only interact with the medium via its editor.

The learning capability that such a forum provides changes fundamentally the notion of learning itself: listeners can form their own understandings and opinions in an unguided manner. At the same time, it provides the necessary guarantees that it does not follow the established standards of a particular learning centre which estimates what has value as knowledge, to whom such knowledge is addressed or what gains one can have from it. Subsequently, the danger of binding the information provider to criteria that specific persons set is eliminated. The multimedia dimension that the Internet supports should not be considered lightly, particularly since it appeals to an ever-increasing audience. This aspect allows the speaker to provide information that directly appeals to the listeners' aesthetics and feelings, like artistic pictures, musical sound, and images of current events that raise emotions. The listener can receive this information at the pace her perception /understanding allows. 


\section{INTERNET: THE WORLDWIDE CAFÉ FOR THE HUMANITIES}

From a different viewpoint, the Internet can be seen as a café. This is a subtle role, one that associates the world wide web with the cafés in the French pre-revolution era. The cafés at that period were the place where the foundations of the French revolution were set. It was the fruitful debates and controversial issues of day-today life that were nursed to become the driving force behind a sequence of events that so dramatically altered the course of a nation.

In the case of the Internet, there are no country borders. The country is the world as a whole. The network becomes the place that nurses a new kind of revolution, one that is not limited to information technology but could address issues such as environmental policies, human rights, etc. The freedom to express one's views and Weltanschauung ${ }^{3}$ [1] in a forum as wide as the Internet is bound to meet controversy and to raise issues that may lead to an uncontrollable boom of this newly found worldwide newspaper. In such a case, it is not beyond the imagination to force a new state of affairs. The role of this service may well prove to be one that could eventually instigate a sociological change.

In any case, whatever the course of things may be, one thing is certain: the face of information technology will dramatically change, changing along the way the manner in which people think and respond to important issues.

The concept of a café, although it has a lot in common with that of the agora, is significantly different. In this case, users do not observe or demonstrate significant and documented views under whatever criteria. Their participation is not considered as that of a simple listener or as a step towards the podium but is one that is continuously involved in debates. Even if users possess unformed opinions, they will form a clearer picture regarding the matter they discuss, they will assign their opinions the appropriate importance, will identify their significance within the broader framework, and will correct misconceptions so that they can convince others or even themselves.

Although the agora and café roles of the Internet can be thought of as very similar, in the context of the former, the speaker is considered to possess the knowledge regarding the topic under discussion. Subsequently, it is up to the speakers' discretion either to involve her audience in the discussion in a dialectic manner, like Socrates did, or simply to express her views in an attempt to convince them, as Demosthenes did. In both cases, the audience's role is a rather passive one, altered only by the speaker's interventions or when a member of the audience who believes can act as a speaker. In the latter, the cafe role of the Internet, speaker and audience interchange roles as circumstances occur. There is no one who is considered knowledgeable. Instead, everyone contributes to the discussion. Agreements or disagreements may occur and through them it is possible for personal opinions to be modified and widely accepted manifestos to be formed. The difference between a café and an agora can be shown when we consider Socrates' sentencing to death and Louis XVI's decapitation. Socrates persuaded his audience that to escape from prison would violate the philosophy and ethics of his teaching. The decapitation of Louis XVI was based on a decision formed as a result of common agreement among the parties involved in the French Revolution.

Despite the fact that this aspect of the Internet's role appears to be a less important one, it is as substantial as the former since it gradually leads listeners to 
realize that they participate in a group, they act according to the group's charter which by the way they can evaluate and criticize, they can obtain a philosophical consciousness not in the passive way of adopting the social norms of the society they live in but through their active participation in the formation of such opinions and the establishment of the personal role they can or must play.

The significance of the conversations in the café is very much like those that take place in everyday life enhanced by the fact that they are not restricted to a particular neighborhood that is influenced by local matters, norms or the preferences of particular people.

Like the cafés in pre-revolution Paris, which were the places where small groups gathered to express opinions and views of what was possible and larger groups were formed as opinions were clarified and defended, so the Internet can play the role of a meeting place for groups of people, irrespective of frontiers and local dependencies, where opinions broad enough to cover matters of world importance can be moulded.

The importance of such discussions for humanity is very high. Through the simple and narrowed range of interventions that occur as a result of the various debates, a holistic and gradually reformed perception regarding any matter will emerge; while, at the same time, misconceptions may arise either by embracing rumours or by distracting reality, present or past, on purpose. Such discussions will enable the formation, and subsequent evaluation, of opinions that are established either because it was not possible to review them or due to lack of debate.

As far as art is concerned and, in particular, local art, through the use of multimedia-enabled communications, the Internet becomes the vehicle that makes such information available worldwide. The identification of its value comes through the exchange of ideas. Seen as an aggregation, it is possible to provide the foreigner with a clearer picture of local, aesthetic characteristics and to provide the local person with the capability of objectively and critically evaluating her personal views on the matter.

\section{INFORMATIONAL FREEDOM: WHAT DO WE DO WITH IT?}

The freedom that the massive information availability could provide with respect to the social, political, and economic aspects of humanity could well lead to chaotic situations. If such a situation occurs, the information provided may become a vehicle for misconceptions and inconsistencies. Furthermore, locating this information may become a difficult and, in some cases, futile exercise for the end user. Even if mechanisms for accurate information location are devised, another issue that arises even under normal conditions is that of information abundance (which can lead to confusing and contradicting knowledge acquisition). Consequently, instead of the user's role being one of exploration of knowledge, this role becomes a struggle to keep up with the information flood.

With respect to the humanities, this can have devastating effects on a person's work, particularly when this work revolves around research and educational matters. Clearly, it is of fundamental importance to ensure the orderly flow of information by providing the appropriate infrastructure and frameworks. 
Such infrastructure and its underlying frameworks must ensure both the use of existing technologies and platforms and, at the same time, lay the foundations for technological development to come [2]. The former is of immense importance, even though some of the technologies currently used are becoming obsolete, such as email services that do not provide multimedia capabilities. Firstly, users who are accustomed to using a particular service, find it difficult in many cases to keep up and at the same time keep track of the ever-emerging latest releases of software and hardware. Secondly, since the area of interest of these users is not in sheer computer science but the role of computers as a means to an end, it is of no value to these users to be constantly on the edge of the technology. Thirdly, investing time and money in technologies that are old within the space of six months to a year is pointless, particularly when one considers latest developments such as the network computer.

The freedom provided by the Internet has been often criticized by the press, with particular focus on the influence it exercises on small children as well as for penetrating one's home. Regarding the latter, one can assert that nothing reaches the user unless the user seeks for it on purpose. Unlike the publicity communicated through television, information available through the Internet cannot in general affect its recipients unless, of course, this is done consciously. Although sinister influences may result, this will happen in cases where people fail to evaluate critically the information they receive.

Consequently, such actions are performed using the user's own judgement and responsibility to a much greater extent than is required when shuffling through a magazine on a bookstand. As far as children are concerned, access to the Internet is only possible if they are given the appropriate means, means provided by the adults responsible for them. Such means are more closely controlled than the currently available yellow press or the danger of being influenced by everyday life events.

The Internet's proper or improper use is very much like the selection of a good or bad movie in the cinema or on television. In truth, it depends on one's culture. Even in this aspect, the Internet can cater for participation and discussion and hence cultural development, a capability that does not provide the unilateral communiqué between the so-called media and the listener. The listener's pseudo- participation in television programmes, like 'call us' programmes, has little to do with the truly in-depth expression of a person's opinion. In such television programmes, the subject is always closed and pre-selected, the reasons for it range from pure advertisement to subtle message-passing, the available time is minimal and, in any case, concerns only a small sample of the audience. If the dangers occurring from the Internet, where children are faced with questions regarding ethics or personal life, are compared to the ones that occur from toy advertising, synthetic food, pink telephone numbers or even those coming from directed information, the criticism that the Internet is faced with can only be moderately explained as impressiongenerating reporting.

The same holds for the humanities except that, in this case, under the pretense of epistemy, nationalistic factors that look on the spread of new ideas as serving particular interests become a serious factor of influence. More often than not such attempts regarding history, cultural heritage or the uniformity and aggregation of nations stem, for the uninformed listener, from dark sources and come to challenge 
historical validity. The Hellenic world is particularly sensitive to this matter since it has already, and is still, experiencing such attempts that unfortunately do not only originate from nationalistic trends in neighbouring countries but also from conscious economic and political policies of foreign countries that are only interested in succeeding in their foreign policy.

The answer in all cases is the same. Inhibition is not the solution to the problem since, despite being ineffective, it can lead to underground sources of information control. By contrast, through uncensored information and juxtaposition of the real facts, the truth will eventually emerge.

The participation of the offended person or group in the agora free-forum and the epistemic objective position are the means that will provide uninformed people with the ability to check, evaluate, and to conclude. Their participation in a cafe will enable them to debate objectively so that they can form the proper and uninfluenced opinions, make the interconnections between facts and other opinions, and assign the appropriate significance to matters. Such opinions will eventually affect and influence the place where decisions that inhibit historical facts take place.

\section{CONCLUSIONS}

The use of the Internet as an agora and as a French pre-revolution café can play an important role in the humanities due to its frontier-less opening in either expressing or observing opinions. The use of the podium as an additional facility, in a virtual interactive book providing insights to a specific knowledge domain which deals with problems concerning the actual world, will enable the user to learn about the subject she wants in a way that guarantees participation and discussion.

Exchange of ideas through the Internet, exploiting different resources in a frontier-less way, permits people to feel as though they are participating in a global village, disposing of the unlimited resources of digital libraries where information is sustained in real-time intervals.

Pupils can participate in that village for peer learning as well as follow important courses and, thus, access for all schools may be assured. This access, however, cannot guarantee participation: participation may be assured by the application of the appropriate didactic methodology, allowing pupils to investigate knowledge by participating in debates, expressing opinions, and undertaking actions according to their own estimations.

The Internet by itself is a digital city. Search and communication is allowed, strongly supported when the person is able to identify her interests. However, there is always a need for supporting person-to-person communication: the huge amount of available information that can be reached leads to a rather impersonal aspect; it may be complemented by the expression of personal opinions, and such opinions cannot be interchanged unless a specific domain for each subject kernel is constructed and made public. So, the agora may be considered rather as a requisite. On the other hand, the café allows the user to participate freely in a community, and thus this is a must rather than a simple possibility. 
Both roles that the Internet can play have significant importance in the largescale proliferation of opinions, resolution, and the rectification of misunderstandings regarding historical, ethnic, and linguistic issues.

In any case, as Sun's ex-Chief Technical Officer, Dr. Schmidt said: 'The Internet was the first technology that we as humans had built that we truly did not understand. We are only beginning to discover its uses. Each time it starts to make sense, it changes. For instance, in that old corporate business model, there was an Intranet - representing the insiders or good guys - and the Internet representing the outside or bad guys. Those two should be together. There should be no division ...'

\section{REFERENCES}

1. Checkland P. B.: The use of the term Weltanschauung in Soft Systems Methodology. Journal of Applied Systems Analysis, Vol. 13, 1986.

2. Theotokis D., Gyftodimos G., and Georgiadis P. Atoms: A Methodology for Component Object Oriented Software Development Applied in the Educational Context. In the Proceedings of the 3rd International Conference on Object Oriented Information Systems, D. Patel, Y. Sun and S. Patel (Eds.), Springer, 1996, pp. 226-242.

1 Learning is considered here within its broader meaning, including that of traditional subject learning.

2 The term communication is used here within its broader meaning of addressing issues of thought exchange and proliferation.

3 The term Weltanschauung refers to one's point of view regarding a situation of concern. 